\title{
HD 85567: A Herbig $B[e]$ star or an interacting $B[e]$ binary?
}

\section{Resolving HD 85567's circumstellar environment with the VLTI and AMBER ${ }^{\star} \star \star \star$}

\author{
H. E. Wheelwright, G. Weigelt, A. Caratti o Garatti, and R. Garcia Lopez
}

\begin{abstract}
Max-Planck-Institut für Radioastronomie, Auf dem Hügel 69, 53121 Bonn, Germany
e-mail: hwheelwright@mpifr-bonn.mpg.de
\end{abstract}

Received 24 June 2013 / Accepted 12 August 2013

\section{ABSTRACT}

\begin{abstract}
Context. HD 85567 is an enigmatic object exhibiting the $\mathrm{B}[\mathrm{e}]$ phenomenon, i.e. an infrared excess and forbidden emission lines in the optical. The object's evolutionary status is uncertain and there are conflicting claims that it is either a young stellar object (YSO) or an evolved, interacting binary.

Aims. To elucidate the reason for the B[e] behaviour of HD 85567, we have observed it with the VLTI and AMBER.

Methods. Our observations were conducted in the $K$-band with moderate spectral resolution $\left(R \sim 1500\right.$, i.e. $\left.200 \mathrm{~km} \mathrm{~s}^{-1}\right)$. The spectrum of HD 85567 exhibits $\mathrm{Br} \gamma$ and $\mathrm{CO}$ overtone bandhead emission. The interferometric data obtained consist of spectrally dispersed visibilities, closure phases and differential phases across these spectral features and the $K$-band continuum.

Results. The closure phase observations do not reveal evidence of asymmetry. The apparent size of HD 85567 in the $K$-band was determined by fitting the visibilities with a ring model. The best fitting radius, $0.8 \pm 0.3 \mathrm{AU}$, is relatively small making HD 85567 undersized in comparison to the size-luminosity relationship based on YSOs of low and intermediate luminosity. This has previously been found to be the case for luminous YSOs, and it has been proposed that this is due to the presence of an optically thick gaseous disc. We demonstrate that the differential phase observations over the $\mathrm{CO}$ bandhead emission are indeed consistent with the presence of a compact ( $\sim 1 \mathrm{AU})$ gaseous disc interior to the dust sublimation radius.

Conclusions. The observations reveal no sign of binarity. However, the data do indicate the presence of a gaseous disc interior to the dust sublimation radius. We conclude that the data are consistent with the hypothesis that HD 85567 is a YSO with an optically thick gaseous disc within a larger dust disc that is being photo-evaporated from the outer edge.
\end{abstract}

Key words. stars: individual: HD 85567 - circumstellar matter - stars: formation - stars: variables: T Tauri, Herbig Ae/Be stars: emission-line, $\mathrm{Be}-$ techniques: interferometric

\section{Introduction}

The formation and early evolution of massive stars is difficult to study and, as a result, still not fully understood. This is partly because sites of massive star formation are typically situated at greater distances than nearby sites of low mass star formation. In addition, massive stars form rapidly, deep within their natal clouds. These factors make detailed study of the small scale environment of young massive stars challenging. Consequently, our understanding of how the star formation process depends on mass is incomplete. To address this issue, it is important to characterise the circumstellar environment of massive young stars and contrast this to the case of low mass star formation.

Most studies on the comparison between low and high mass star formation have focused on Herbig Ae/Be stars. These objects are pre-main-sequence objects identified by the presence of an infrared excess and emission lines and have a mass of 2-15 $M_{\odot}$ (Herbig 1960; Thé et al. 1994). Herbig Ae/Be $(\mathrm{HAe} / \mathrm{Be})$ stars span the transition from low to high stellar masses. Since they are optically visible and relatively luminous, $\mathrm{HAe} / \mathrm{Be}$ stars offer an opportunity to study the circumstellar

* Based on observations conducted at the European Southern Observatory, Paranal, Chile, which were obtained via the program 089.C-0220.

$\star \star$ Appendices are available in electronic form at

http://www. aanda.org geometry of young stellar objects (YSOs) at intermediate and high luminosities. As a result, there have been many studies of the circumstellar environment of $\mathrm{HAe} / \mathrm{Be}$ stars (see e.g. Meeus et al. 2001; Natta et al. 2001; Vink et al. 2002; Millan-Gabet et al. 2001; Eisner et al. 2004; Acke et al. 2005; Monnier 2005; Kraus et al. 2008a,b; Weigelt et al. 2011). An extensive overview of the structure of the inner discs of Herbig $\mathrm{Ae} / \mathrm{Be}$ stars is presented in Dullemond \& Monnier (2010). Here, we focus on the differences between the circumstellar environments of Herbig Ae (HAe) and Herbig Be (HBe) stars.

Based on the analysis of the infrared excesses of such objects, Meeus et al. (2001) suggest that HAe/Be stars can be split into two Groups: I and II. It has been proposed that these two groups represent different disc geometries. Group I objects, objects with prominent mid infrared excesses, are thought to possess flared discs. Group II objects, which have less strong excesses in the mid infrared, are thought to possess flatter disc geometries. While the majority of HAe stars are classified as Group I objects, HBe stars generally belong to Group II (Acke et al. 2005). Whether this dichotomy is due to the more rapid evolution of luminous YSOs, a consequence of the dependence of disc geometry on the temperature of the central star or a combination of these and other factors is not clear.

Another difference between the discs around HAe and HBe objects was discovered with long baseline interferometry in the infrared. In the past decade, the circumstellar environments 
of many $\mathrm{HAe} / \mathrm{Be}$ objects have been spatially resolved by interferometric observations in the near infrared (see e.g. MillanGabet et al. 1999, 2001; Eisner et al. 2003, 2004, 2007, 2009, 2010; Monnier 2005; Monnier et al. 2006; Malbet et al. 2007; Kraus et al. 2008b; Tannirkulam et al. 2008; Benisty et al. 2010; Renard et al. 2010; Kreplin et al. 2012). It has been noted that low and intermediate luminosity HAe and HBe objects follow a tight correlation between their size in the $K$-band and their luminosity. However, it has also been found that the most luminous $\mathrm{HBe}$ objects appear undersized based on this relationship (Monnier 2005). It has been proposed that this could be due to the presence of an optically thick inner disc that shields the outer dust disc from stellar radiation, allowing it to exist closer to the central star (Monnier \& Millan-Gabet 2002). This inner disc may represent an optically thick accretion disc, and this hypothesis is consistent with broad-band, long baseline interferometry in the infrared (Monnier 2005; Vinković \& Jurkić 2007). Furthermore, such discs can provide a significant contribution to the NIR excess of their host star. Since this emission originates from within the dust sublimation radius, it will also contribute to the object appearing undersized. This hypothesis has been shown to be valid in a few cases (see Kraus et al. 2008b). However, since only a few high luminosity $\mathrm{HBe}$ stars have been studied with high spatial resolution, the structure and evolution of their discs is still not fully understood. Indeed, it is still not clear whether or not all luminous YSOs are undersized (see e.g. Dullemond \& Monnier 2010).

The issue is further complicated by the fact that it can be difficult to determine the evolutionary status of luminous emission line objects. Several objects that may be Herbig Be stars could also be evolved objects (see e.g. Kraus et al. 2009). The uncertain evolutionary status of such objects makes it difficult to obtain an overview of the circumstellar environment of luminous YSOs.

To address these issues, we observed the Herbig Be candidate HD 85567 using the VLTI and AMBER. HD 85567 (CPD $-60^{\circ} 1510$, Hen $3-331$ ) is a luminous B[e] object of uncertain evolutionary status. It listed as a Herbig Be star with a spectral type B5Ve by Thé et al. (1994). The object was also listed as a HAeB[e] star, i.e. a Herbig Be star that exhibits forbidden line emission, by Lamers et al. (1998). Based on the object's infrared SED, Malfait et al. (1998) classify HD 85567 as an object with a double dust disc. However, the lack of a significant dip between the near and mid infrared flux indicates that the disc of HD 85567 is relatively un-evolved. Recently, Verhoeff et al. (2012) included HD 85567 in a sample of HAeBe stars observed in the $N$-band. These authors classify the SED of HD 85567 as that of a type II object using the scheme of Meeus et al. (2001). This could indicate the presence of an optically thick inner disc that prevents the outer disc from flaring.

The numerous studies mentioned above classify HD 85567 as a relatively luminous $\left(L_{\star} \sim 15000 L_{\odot}\right)$ YSO. However, there is an alternative scenario. Miroshnichenko et al. (2001) note that HD 85567 does not exhibit a prominent far infrared excess. These authors suggest that this could indicate that this object is not a YSO and that the presence of warm dust in its environment might be attributed to mass loss driven by binary interactions. However, this is only conjecture as these authors do not find direct evidence of a companion. HD 85567 was later classified as a binary by Baines et al. (2006). These authors present a clear photo-centre shift of 29 milli-arcsec to the south over the $\mathrm{H} \alpha$ line. Assuming that the flux ratio in the optical is unity and that only one component exhibits $\mathrm{H} \alpha$ emission, this implies a separation of around 60 milli-arcsec (mas), approximately $100 \mathrm{AU}$ at $1.5 \mathrm{kpc}$. However, it is likely the optical flux ratio is not unity and thus that the separation is many times larger. Therefore, it is not clear if the companion detected is close enough to interact with the primary, thus causing the $\mathrm{B}[\mathrm{e}]$ behaviour of this object. As a result, at present, it is still not clear whether HD 85567 is a bone fide YSO or an interacting $\mathrm{B}[\mathrm{e}]$ binary.

High resolution observations have the potential to address this issue by examining whether HD 85567 has an additional companion at a smaller separation. Furthermore, high resolution observations also provide an opportunity to probe the structure of the object's disc. With this in mind, we observed HD 85567 with the VLTI and AMBER. This paper presents the observations and is structured as follows. The observations are presented in Sect. 2. The results are presented in Sect. 3 and discussed in Sect. 4. The paper is concluded in Sect. 5.

\section{Observations and data reduction}

HD 85567 was observed with the VLTI and AMBER in the $K$-band using the medium spectral resolution mode. This provides a spectral resolution of $R \sim 1500$ or $\Delta v \sim 200 \mathrm{~km} \mathrm{~s}^{-1}$ and a wavelength coverage of $\sim 2.15-2.45 \mu \mathrm{m}$. Observations were conducted using the UT1-UT2-UT3 telescopes on two occasions and the UT2-UT3-UT4 telescopes on two additional occasions. In all cases, FINITO (Gai et al. 2004) was used to provide fringe tracking. The observations span a period of approximately 11 months. In all cases, observations of HD $85567(H=6.7$, $K=5.8)$ were conducted between observations of the calibrator objects HD $85313(H=5.3, K=5.1)$ and HD $84177(H=5.4$, $K=5.3)$. The projected baselines are displayed in Fig. 1 and a $\log$ of the observations is presented in Table 1.

The data were reduced in the standard fashion for AMBER data using the JMMC amdlib package ${ }^{1}$ (see Tatulli et al. 2007b; Chelli et al. 2009). A variety of selection rates were used to choose frames of the interferograms for processing. Accurate visibilities require a low selection rate, as a low signal-to-noise $(\mathrm{S} / \mathrm{N})$ can bias the results, while precise differential phases can benefit from relatively high selection rates. They are not biased in the same way and thus the precision can be increased by increasing the amount of frames selected. Calibration of the data, visibilities and closure phases, was performed using a transfer function constructed from the observations of the calibrators. The transfer functions were constructed assuming that the two calibrators, HD 84177 and HD 85313, can be described as uniform discs with radii given by $0.435 \pm 0.031$ and $0.449 \pm 0.032$ mas respectively (Bonneau et al. 2006, 2011).

Comparison of the transfer functions and observations of HD 85567 (shown in Fig. A.1) reveals an apparent change in the appearance of the target. In two cases (2012/04/06 and 2013/03/04), the visibilities of HD 85567 are the same as the calibrators, indicating a compact source. In the other two cases (2012/05/06 and 2012/12/29), the visibilities of HD 85567 are noticeably lower than the calibrators, indicating an extended source. If this behaviour were real, this would indicate that the environment of HD 85567 was compact at the beginning of our observations, became extended and then returned to its initial appearance.

The simplest explanation of this behaviour is that HD 85567 has a previously undetected binary companion and the period of the system is of the order of approximately 1 year. However, the lack of a strong closure phase signal is not consistent with

Version 3.0.3, available at http://www . jmmc . fr/amberdrs 
Table 1. Log of the series of AMBER observations.

\begin{tabular}{lcccccc}
\hline \hline Object & $\begin{array}{c}\text { Seeing } \\
\left({ }^{\prime \prime}\right)\end{array}$ & $\begin{array}{c}\text { Coherence time } \\
(\mathrm{ms})\end{array}$ & $\begin{array}{c}\text { DIT } \\
(\mathrm{ms})\end{array}$ & Telescopes & $\begin{array}{c}\text { Baselines } \\
(\mathrm{m})\end{array}$ & $\begin{array}{c}\text { PAs } \\
\left({ }^{\circ}\right)\end{array}$ \\
\hline $\mathbf{2 0 1 2 / 0 4 / 0 6}$ & & & & & & \\
HD 84177 & 0.81 & 2.98 & 500.0 & UT1-UT2-UT3 & $50.0 / 43.3 / 92.6$ & $23.8 / 37.7 / 30.3$ \\
HD 85567 & 0.78 & 3.10 & 500.0 & UT1-UT2-UT3 & $48.4 / 42.1 / 89.8$ & $28.5 / 43.1 / 35.2$ \\
HD 85313 & 0.78 & 3.11 & 500.0 & UT1-UT2-UT3 & $48.0 / 41.5 / 88.7$ & $32.1 / 47.3 / 39.1$ \\
$\mathbf{2 0 1 2 / 0 5 / 0 6}$ & & & & & & \\
HD 84177 & 0.88 & 5.60 & 500.0 & UT2-UT3-UT4 & $41.8 / 89.0 / 61.3$ & $226.7 / 84.2 / 108.8$ \\
HD 85567 & 1.06 & 4.95 & 500.0 & UT2-UT3-UT4 & $40.5 / 88.3 / 61.8$ & $231.7 / 89.8 / 113.6$ \\
HD 85313 & 0.55 & 8.52 & 500.0 & UT2-UT3-UT4 & $37.6 / 85.5 / 62.5$ & $242.9 / 104.4 / 127.9$ \\
$\mathbf{2 0 1 2 / 1 2 / 2 9}$ & & & & & & \\
HD 84177 & 0.55 & 4.11 & 500.0 & UT2-UT3-UT4 & $44.5 / 88.4 / 56.1$ & $205.7 / 58.2 / 83.4$ \\
HD 85567 & 0.52 & 4.35 & 500.0 & UT2-UT3-UT4 & $44.0 / 88.9 / 56.9$ & $208.2 / 60.7 / 85.3$ \\
HD 85313 & 0.70 & 3.31 & 500.0 & UT2-UT3-UT4 & $43.8 / 89.2 / 58.0$ & $211.9 / 65.6 / 90.3$ \\
$\mathbf{2 0 1 3 / 0 3 / 0 4}$ & & & & & & \\
HD 84177 & 0.86 & 4.33 & 500.0 & UT1-UT2-UT3 & $50.0 / 43.3 / 92.6$ & $23.7 / 37.6 / 30.1$ \\
HD 85567 & 0.55 & 5.80 & 500.0 & UT1-UT2-UT3 & $48.9 / 42.6 / 90.8$ & $25.8 / 40.0 / 32.4$ \\
HD 85313 & 0.54 & 6.64 & 500.0 & UT1-UT2-UT3 & $48.7 / 42.2 / 90.1$ & $28.9 / 43.6 / 35.7$ \\
\hline
\end{tabular}

Notes. ${ }^{(1)}$ DIT represents the detector integration time.

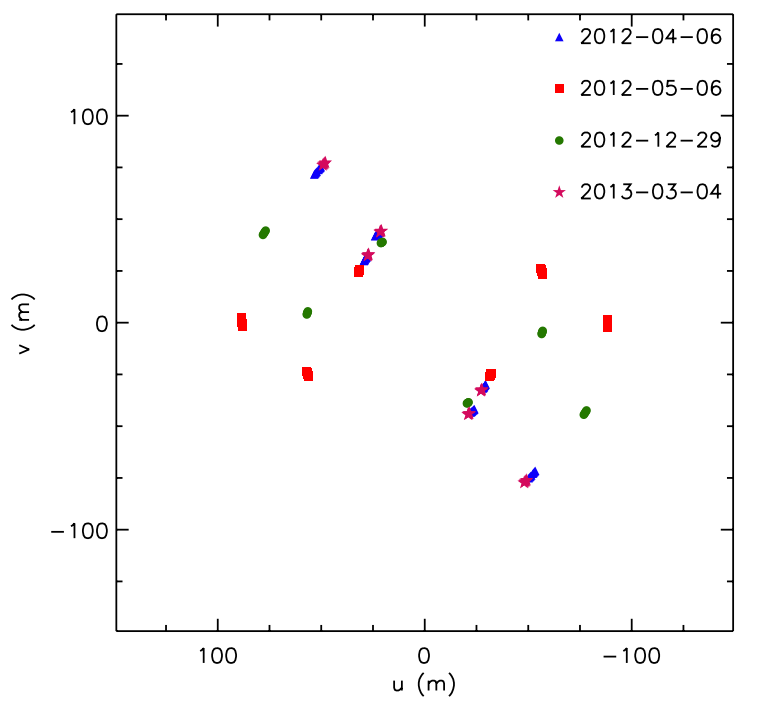

Fig. 1. Projected baselines of the observations of HD 85567.

this scenario. Therefore, we explored the possibility that an observational bias is affecting the visibilities (this is discussed in Appendix B).

It was found that when the target visibilities are significantly lower than those of the calibrators, there is a marked difference in the distributions of ratio of the target and calibrator fringe $\mathrm{S} / \mathrm{N}$. The $\mathrm{S} / \mathrm{N}$ associated with the fringes is the $\mathrm{S} / \mathrm{N}$ of the coherent flux, and is an important quantity to consider in the reduction of AMBER data (Tatulli et al. 2007a). We surmise that the difference between the target and calibrator observations on these dates is a bias caused by the fringe tracking performance degrading when observing the target. This is supported by the FINITO data recorded by the RMNREC software. The degradation of FINITO's performance when observing the target was likely due to two reasons. Firstly, the target is a magnitude fainter than the calibrators in the $H$-band where fringe tracking is conducted. Secondly, the science observations were associated with poor seeing (especially on 2012-05-06). It is surmised that on the dates in question, poor fringe tracking resulted in an artificially lower fringe contrast for the observations of HD 85567, when compared to the calibrator observations. Consequently, only the observations when the fringe $\mathrm{S} / \mathrm{N}$ distributions of the target and calibrator observations are similar can be calibrated. In principle, the observations of 2012-04-06 and 2013-03-04 offer reliable calibration. However, since the fringe S/Ns of the observations conducted 2012-04-06 are relatively low, the rest of the paper focuses exclusively on the observations obtained on the date 2013-03-04. These data were taken after AMBER's performance was improved in January 2013 and thus both the target and calibrator observations exhibit relatively high fringe S/Ns (see Fig. B.1).

\section{Results}

The interferometric observations of HD 85567 conducted on 2013-03-04 are presented in Fig. 2. The time averaged closure phase is close to zero. We conclude that there is no compelling evidence that the environment of HD 85567 is asymmetric on the scales probed by these observations. The calibrated visibilities are relatively high, $\sim 0.7-0.8$. This indicates that the environment of HD 85567 is only marginally resolved. To determine the characteristic size of the continuum emission region, the calibrated visibilities were fit with a geometric ring model. This is discussed in Sect. 3.1.

The $K$-band spectrum of HD 85567 exhibits $\mathrm{Br} \gamma$ and CO first overtone bandhead emission. The differential visibilities and phases across the $\mathrm{Br} \gamma$ and $\mathrm{CO}$ overtone emission are presented in Fig. 3. In both cases, no conspicuous signature is observed. This suggests that the distributions of the continuum, $\mathrm{Br} \gamma$ and $\mathrm{CO}$ overtone emission are similar. However, it is possible a slight change occurs in the differential phases associated with the longest baseline over the $\mathrm{CO}$ bandhead emission. This is discussed in more detail in Sect. 3.2.

\subsection{Ring model}

To fit the visibilities, the ratio of the infrared excess and photospheric emission was determined. This was achieved by analysing the SED of HD 85567, which was constructed by taking data from the literature and using the VOSA utility (Bayo et al. 2008). The SED contains data from 2MASS 

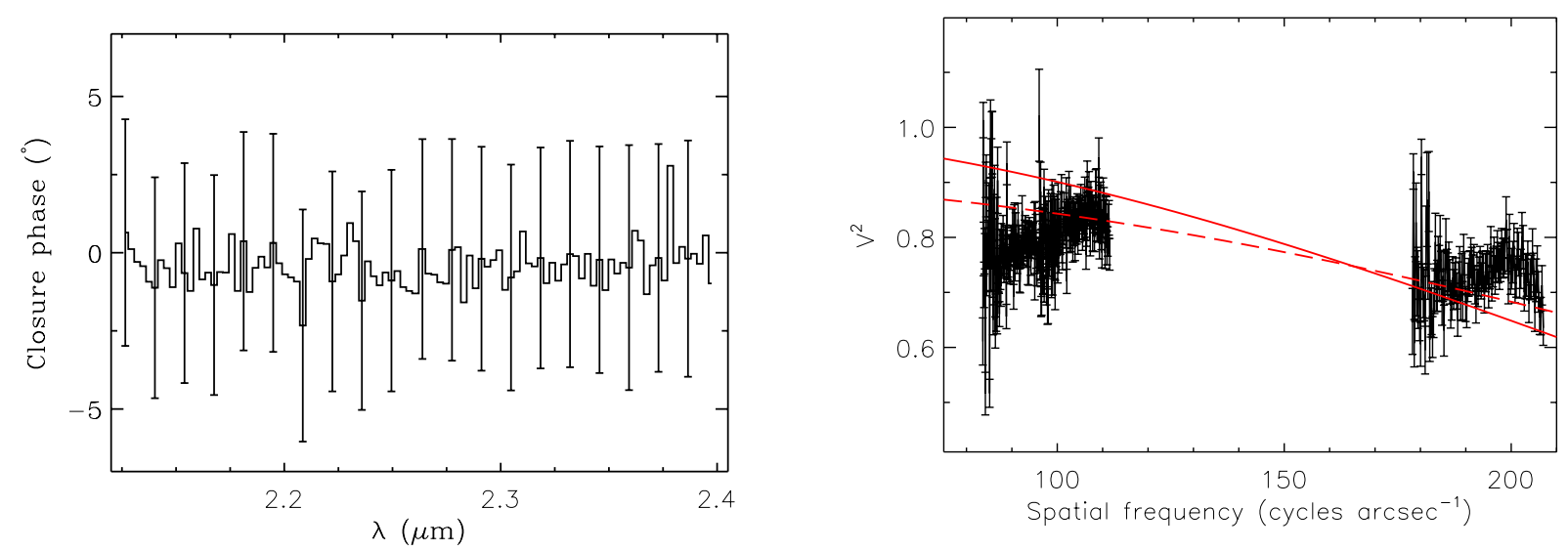

Fig. 2. Time-averaged closure phase and squared visibility observations of HD 85567. The panel on the left presents the closure phases. The closure phase error bars shown represent the mean error in the measurements. A frame selection of 80 per cent was used. In the panel on the right, we present the squared visibilities. A frame selection of 20 per cent was used. The errors represent the mean error in the calibrated visibilities. The solid line is the visibility profile of a ring with a radius of 0.69 mas, $1.0 \mathrm{AU}$ at $1.5 \mathrm{kpc}$. The long-dashed line corresponds to a ring with a radius of 0.56 mas $(0.8 \mathrm{AU})$ with the addition of a background that accounts for 5 percent of the total flux.

Table 2. Adopted stellar parameters.

\begin{tabular}{lcc}
\hline \hline Parameter & Value & Ref. \\
\hline Spec. Typ. & $\mathrm{B} 2$ & $\mathrm{M} 01$ \\
$T_{\text {eff }}$ & $19000 \mathrm{~K}$ & $\mathrm{M} 01$ \\
$d$ & $1.5 \pm 0.5 \mathrm{kpc}$ & $\mathrm{M} 01$ \\
$R_{\star}$ & $9 \pm 2 R_{\odot}$ & $\mathrm{V} 12$ \\
$A_{V}$ & $1.1 \pm 0.1$ & $\mathrm{~V} 12$ \\
$\log L_{\star}$ & $4.17 \pm 0.16 L_{\odot}$ & $\mathrm{V} 12$ \\
$M_{\star}$ & $12 \pm 2 M_{\odot}$ & $\mathrm{V} 12$ \\
\hline
\end{tabular}

Notes. M01: Miroshnichenko et al. (2001), V12: Verhoeff et al. (2012).

(Skrutskie et al. 2006), AKARI (Ishihara et al. 2010; Murakami et al. 2007; Onaka et al. 2007), DENIS (DENIS Consortium 2005), IRAS (Beichman et al. 1988; Helou \& Walker 1988), Tycho-2 (Høg et al. 2000), WISE (Wright et al. 2010; Cutri \& et al. 2012), de Winter et al. (2001), Klare \& Neckel (1977), (Schild et al. 1983), Miroshnichenko et al. (2001), Mermilliod \& Mermilliod (1994) and Verhoeff et al. (2012). The data were de-reddened using the extinction relationship of Cardelli et al. (1989) and values of $A_{V}=1.1, R_{V}=3.1$ (see Table 2). The final SED is shown in Fig. 4.

To determine the ratio of the stellar and circumstellar emission, we compared the observed SED to the stellar flux expected for the spectral type of HD 85567. The stellar parameters taken from the literature, including spectral type and effective temperature, are listed in Table 2. Assuming an effective temperature of $T_{\text {eff }}=19000$, the ratio of the excess to stellar emission in the $K$-band is 9.0 .

The visibilities were then fit with a model of a ring, which was assumed to be face on as the data do not show PA-related $V^{2}$ variations indicative of an asymmetric object. The best fit ring radius was found to be given by $r=0.69_{-0.23}^{+0.20}$ mas, which resulted in a minimum chi squared value of $\chi_{R}^{2}=3.59$ (using the rms of the visibility measurements). It was found that the fit could be improved by adding a resolved background component. The minimum contribution from a totally resolved background flux that resulted in a $\chi^{2}<2$ was determined to be approximately 5 percent of the total flux. The resulting best fit ring radius was $r=0.56_{-0.20}^{+0.16}$ mas, which resulted in a minimum chi squared value of $\chi_{R}^{2}=1.68$. The best fit visibility distributions are displayed in the right panel of Fig. 2.

\subsection{Differential visibilities and phases}

The differential visibilities across the $\mathrm{B} \gamma$ line and $\mathrm{CO}$ bandhead emission are presented in Fig. 3. No clear change in visibilities is observed across the $\operatorname{Br} \gamma$ line. There are some suggestions of an increase in visibilities over the line, indicating a compact line emitting region. However, these are not considered significant given the lack of consistency of the position of these increases with respect to the line centre. Since baselines with similar lengths and position angles (UT1-UT2 and UT2-UT3) exhibit different changes in visibility in the approximate region of the $\mathrm{Br} \gamma$ line, the features observed are considered artifacts. The differential visibilities over the CO bandhead emission exhibit several artificial changes across telluric absorption lines. These make it challenging to detect changes in the visibilities across the $\mathrm{CO}$ bandhead emission.

The differential phases across the $\mathrm{Br} \gamma$ and $\mathrm{CO}$ bandhead emission are also presented in Fig. 3. In the case of one baseline (UT1-UT2), it appears that there is a change in phase across the $\mathrm{Br} \gamma$ line. The behaviour of the phase variation with wavelength; a negative change on the blue side of the line and a positive change over the red side, is similar to that expected in the case of line emission originating in a rotating medium. However, since the phases associated with the similar UT2-UT3 baseline (49 and $43 \mathrm{~m}$ at PAs of 26 and $40^{\circ}$ respectively) do not exhibit this behaviour, it is suggested that the phase signal discussed is also an artifact. In general, no prominent offset is observed in the differential phases across the $\mathrm{CO}$ bandhead. However, since the observed spectrum features several $\mathrm{CO}$ overtone transitions, we could increase the precision of the differential phase observations by co-adding the data across the individual transitions. This was done using the data associated with the longest baseline (UT3-UT1, $91 \mathrm{~m}$ ) as these observations access the smallest scales. The results are discussed in the following section (3.2.1).

\subsubsection{Photo-centre offset over the $\mathrm{CO}$ bandhead emission}

To increase the precision of the differential phase observations obtained with the UT3-UT1 baseline, the phases across the 3 first 

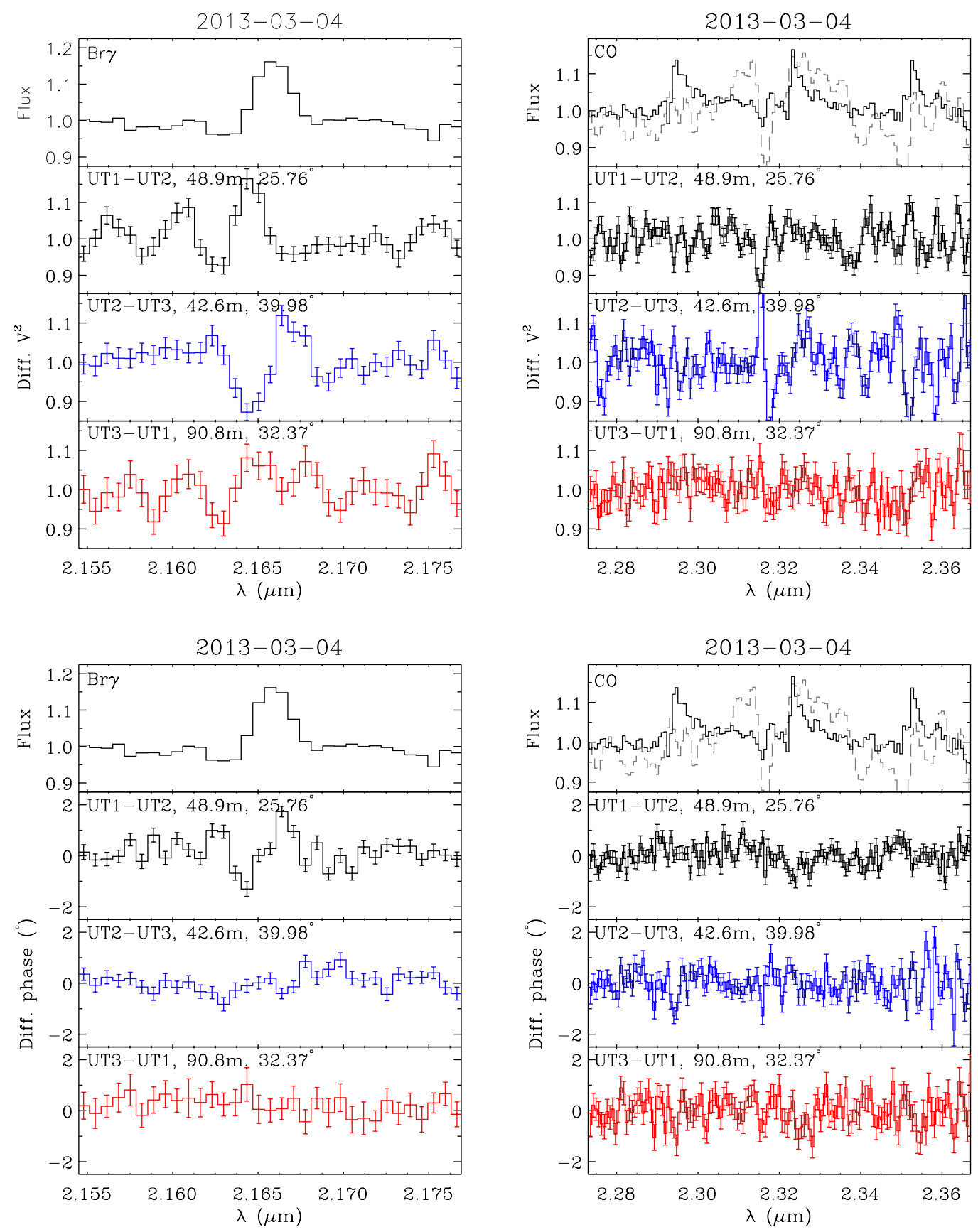

Fig. 3. Time-averaged differential squared visibility (top) and differential phases (bottom) around the Bry line (left) and CO first overtone bandhead emission (right). Error bars shown represent statistical errors on the mean. A frame selection of 20 per cent was used for the visibilities and a selection rate of 80 percent was employed to obtain the averaged phases. The individual AMBER files were merged before frame selection was conducted. The dashed line alongside the $\mathrm{CO}$ emission represents the spectrum before telluric correction.

bandhead transitions were averaged. The photo-centre offset associated with the resultant differential phase signal was calculated using $p=-\frac{\phi}{2 \pi} \frac{\dot{\lambda}}{B}$, where $B$ is the baseline length and $p$ represents the projection of the $2 \mathrm{D}$ photo-centre along the orientation of the baseline. The result is shown in Fig. 5. The observations are consistent with a small offset corresponding to approximately $10 \mu$ as occurring over the bandhead profile. In contrast, offsets larger than approximately $10 \mu$ as can be excluded. Whether this can be used to constrain the location of the $\mathrm{CO}$ bandhead emission was then explored using the model developed in Wheelwright et al. (2010, 2012).

To reduce the running time of the model, it is assumed that the average photo-centre shift associated with the first three
CO overtone transitions $(2 \rightarrow 0,3 \rightarrow 1$ and $4 \rightarrow 2$ ) could be modelled as the shift over the first bandhead $(2 \rightarrow 0)$. This is a simplification but ultimately, the emission of the different bandheads will originate from the same location. Based on the excitations requirements of the different transitions, this approach will slightly over-estimate the average offset. However, given the $0.5 \mathrm{kpc}$ uncertainty in the distance to HD 85567, this was not considered significant.

To calculate the photo-centre offsets associated with CO bandhead emission from a circumstellar disc, we used the model presented in Wheelwright et al. (2012) and the stellar parameters presented in Table 2. The source of the CO emission was represented by a Keplerian disc with power laws describing 


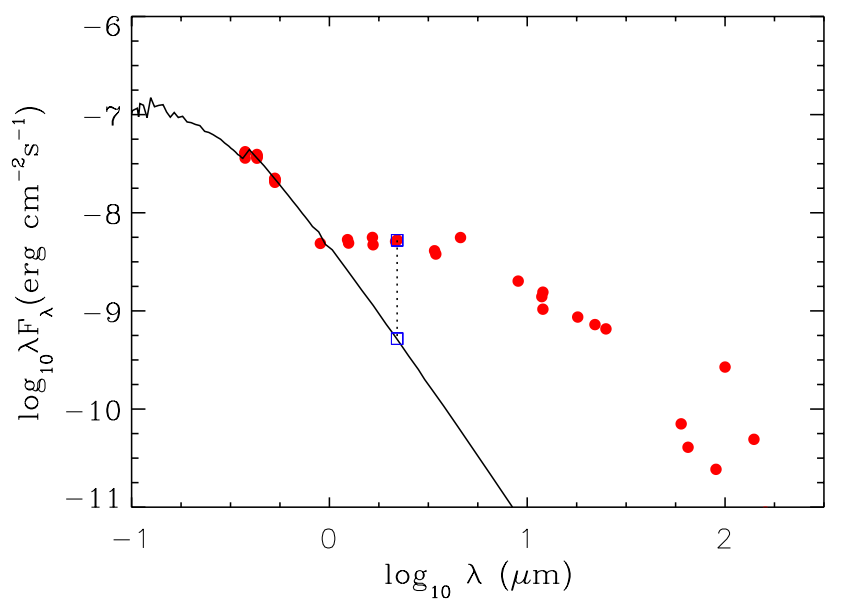

Fig. 4. HD 85567's SED. The data were de-reddened using an $A_{V}=1.1$, and are shown with the predicted SED for the parameters $T_{\text {eff }}=19000$ and $\log g=3.5$.

the radial dependence of the excitation temperature and surface number density. The exponents of the respective power laws were set to $p=-0.5$ and $q=-1.5$. Finally, the inclination was set to $i=35^{\circ}$, which is based on fits to the CO bandhead emission presented in Ilee (2013). It is noted that this value is relatively uncertain as it was derived from a model fit to spectra of moderate, rather than high, spectral resolution. Nonetheless, it serves as a representative value and is sufficient for our purposes. Once the images of the disc at various wavelengths had been calculated, the associated offset was determined from the photo-centre of each image.

We calculated the photo-centre offsets for two models. The first with a relatively small inner radius, $5 R_{\star}$, and a compact outer radius of $1 \mathrm{AU}$, as predicted by the scenario of an optically thick gas disc interior to the dust sublimation radius. The second model featured a larger inner radius, $10 R_{\star}$, and a more extended outer radius of $4 \mathrm{AU}$. This outer radius corresponds to the scenario of an optically thin inner disc and a dust sublimation radius that reproduces the size luminosity relationship of intermediate and low luminosity objects.

The model photo-centre offsets are displayed in Fig. 5. Clearly, the significance of the slight offset observed is low, the tentatively identified signature is approximately 4 times the continuum rms. However, it is evident that the data are consistent with the offset associated with the smaller disc. Furthermore, the data favour the smaller disc over the larger disc as the more extended disc results in an offset that is larger than that observed.

\section{Discussion}

This paper presents new VLTI/AMBER observations of the B[e] star HD 85567. Two scenarios have been proposed to explain the $\mathrm{B}[\mathrm{e}]$ behaviour of this object. One scenario that explains the object's infrared excess and line emission is that it is a YSO with a circumstellar accretion disc. The alternative scenario is that HD 85567 is an interacting binary with circumstellar material that has been deposited though mass loss driven by binary interactions. Here, we discuss our findings in the context of these two scenarios. We also briefly discuss the structure of HD 85567's circumstellar material and consider how this is evolving.

We note that our moderate spectral resolution observations reveal that HD 85567 exhibits ${ }^{12} \mathrm{CO}$ bandhead emission, but not ${ }^{13} \mathrm{CO}$ bandhead emission. In principle, the fact that the

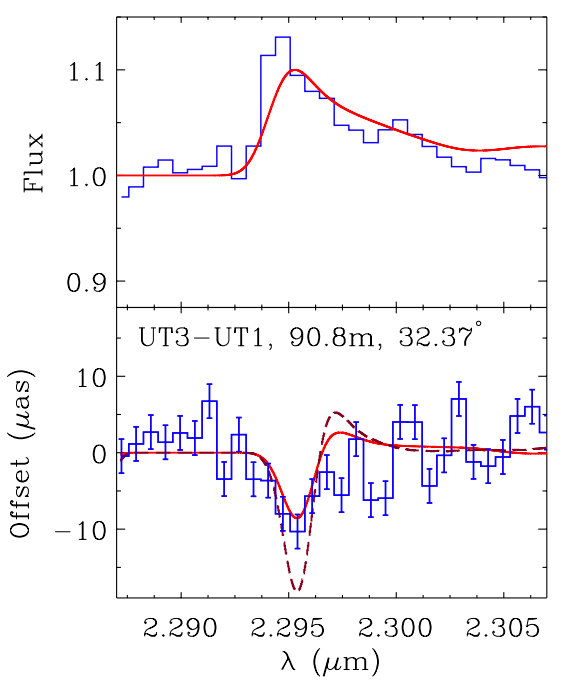

Fig. 5. Photo-centre offsets associated with the $\mathrm{CO}$ bandhead emission. This figure was constructed by co-adding the time averaged differential phase observations obtained on the date 2013-03-04 using the UT1-UT3 baseline across the first $3 \mathrm{CO}$ bandheads. The uncertainty in the differential phase is represented by the rms of the continuum measurements. The smaller model offset (solid line) corresponds to an inner disc radius of 5 stellar radii $(0.2 \mathrm{AU})$ and an outer radius of $1.0 \mathrm{AU}$ ( 0.7 mas). The larger offset (dashed line) was calculated for an inner radius of 10 stellar radii $(0.4 \mathrm{AU})$ and an outer radius of $4.0 \mathrm{AU}$ (2.7 mas, sizes calculated assuming a distance of $1.5 \mathrm{kpc}$ ).

circumstellar material of HD 85567 is not significantly enriched in ${ }^{13} \mathrm{CO}$ favours the YSO scenario (Kraus et al. 2009). However, we note that while the spectrum excludes ratios of ${ }^{12} \mathrm{CO} /{ }^{13} \mathrm{CO}$ below approximately 15 , this is not sufficient to place strong constraints on the evolutionary status of HD 85567 (Kraus et al. 2009).

The closure phase observations provide an additional means to investigate the interacting binary hypothesis. HD 85567 has already been shown to be a binary, although the estimated minimum separation is $\sim 100 \mathrm{AU}$ (and likely many times this, Baines et al. 2006). Since this companion may be too distant to induce mass loss from the primary, we used our high resolution observations to investigate the hypothesis that HD 85567 has an additional, closer companion within the field of view of the UT telescopes (60 mas, $\sim 100 \mathrm{AU}$ ). Since no closure phase signature is detected, the observations do not reveal an additional close binary companion. For completeness, we note that the $u, v$ coverage of the observations discussed is relatively linear. In principle, a companion could escape detection if it was aligned perpendicularly to the projected baselines. However, the additional closure phases associated with the nights of degraded FINITO performance are also consistent with zero, and thus indicate a symmetric source. This is a robust result as a bias in visibilities will not affect closure phase measurements. Therefore, the data support the conclusion that HD 85567 does not appear to have a close binary companion, although a faint companion could still escape detection. We now investigate whether the data are consistent with the hypothesis that HD 85567 is a YSO.

The observed visibilities are relatively high and can be reasonably reproduced using a point source and a ring model. We report that the apparent radius of the $K$-band continuum emitting region of HD 85567 is $r=0.56_{-0.20}^{+0.16}$ mas $(\sim 0.8 \pm 0.3 \mathrm{AU})$. Based on the luminosity of HD 85567 and the predicted dust sublimation radius when the inner disc is optically thin, the expected ring radius is $4.2 \mathrm{AU}$. Therefore, this is considerably smaller 
than expected based on the size luminosity relationship exhibited by YSOs of low and intermediate luminosity (Monnier 2005). This is a robust result as it is most likely independent of a possible bias in the calibrated visibilities due to the use of FINITO. As discussed previously, FINITO can bias the target visibilities to low values. Therefore, if the data are biased, the true size of HD 85567 may be smaller, but not larger. Furthermore, HD 85567 appears undersized even when allowing for the uncertainties in its distance and luminosity (both approximately 30 percent). The undersized appearance of HD 85567 is similar to the case of luminous YSOs. For example, the Herbig Be star V1685 Cyg has a luminosity of $21400 L_{\odot}$ and a $K$-band ring fit radius of $2.15_{-0.18}^{+0.23} \mathrm{AU}$, making it undersized by nearly $3 \mathrm{AU}$ (Monnier 2005). This was also reported to be the case for the early B type Herbig Be star MWC 297 (Weigelt et al. 2011). Therefore, the size of HD 85567 supports the hypothesis that this object is also a YSO.

It has been proposed that the reason for the small sizes of luminous YSO is that their inner discs are optically thick, shielding the inner rim of the dust disc from stellar radiation. This can allow the dust sublimation radius to be located closer to the central star than would otherwise be the case. The optically thick inner gas disc is associated with active accretion discs interior to the dust sublimation radius (Eisner et al. 2004; Monnier 2005). Here we explore whether this scenario is applicable to HD 85567. By considering the combined effect of stellar irradiation and viscous heating, Millan-Gabet et al. (2001) present the temperature of an accretion disc as a function of radius. The equations used are the following:

$T(r)=\left(T_{\mathrm{rep}}^{4}+T_{\mathrm{acc}}^{4}\right)^{\frac{1}{4}}$

in combination with

$T_{\text {rep }}=T_{\star}\left(\frac{1}{3}^{\frac{1}{4}}\right)\left(\frac{R \star}{r}^{\frac{3}{4}}\right)$

and

$T_{\mathrm{acc}}=\left(\frac{3 G M_{\star} \dot{M}_{\mathrm{acc}}}{8 \pi \sigma}\right) r^{\frac{-3}{4}}$

where $\sigma$ is the Stefan-Boltzmann constant, $G$ is the gravitational constant and $\dot{M}_{\text {acc }}$ is the accretion rate. These equations can be used to crudely estimate the expected size of accretion discs by determining the radius where the temperature falls to $1500 \mathrm{~K}$, i.e. the approximate dust sublimation radius.

It has been estimated that HD 85567 accretes material at a rate of rate of approximately $1 \times 10^{-6} M_{\odot} \mathrm{yr}^{-1}$ (based on the object's Br $\gamma$ emission, Ilee 2013). This should be sufficient to ensure an optically thick inner disc (see e.g. Weigelt et al. 2011). Using this accretion rate and the parameters in Table 2, we obtain a predicted dust sublimation radius of $0.9 \mathrm{AU}$. This is consistent with the best fitting ring radius of $0.8 \pm 0.3 \mathrm{AU}$. Therefore, it is certainly plausible that the size of HD 85567 in the $K$-band reflects the presence of an optically thick disc interior to the dust sublimation radius. This is supported by the finding that a gaseous disc $1 \mathrm{AU}$ in size is consistent with the differential phase observations over the $\mathrm{CO}$ bandhead emission. Gaseous discs with radii in excess of $4 \mathrm{AU}$, the location of the dust sublimation radius in the case of an optically thin inner disc, do not reproduce the data well.

We conclude that the observations are consistent with the hypothesis that HD 85567 is a YSO while they do not support the interacting, evolved binary scenario. We find that HD 85567 appears undersized according to the size luminosity relationship of YSOs and demonstrate that this could be due to the presence of an optically thick gaseous disc interior to the dust sublimation radius. Finally, we note that the presence of an optically thick inner disc and the absence of a far infrared excess suggest that HD 85567 is photo-evaporating its disc from the outside. This supports the hypothesis that this is the fate of discs around Herbig Be stars (Alonso-Albi et al. 2009; Verhoeff et al. 2012).

\section{Conclusion}

This paper presents new VLTI/AMBER observations of the enigmatic $\mathrm{B}[\mathrm{e}]$ object HD 85567 . Here we reiterate the salient results.

The object's environment appears compact and symmetric on scales of a few to $100 \mathrm{AU}$. This does not support the hypothesis that the object is an evolved, interacting binary. The apparent radius of HD 85567's environment in the $K$-band is found to be $r=0.56_{-0.20}^{+0.16}$ mas $(\sim 0.8 \pm 0.3 \mathrm{AU})$. This makes the object undersized according to the size luminosity relationship based on YSOs of low and intermediate luminosity. This has previously been found to be the case for luminous YSO and thus the size of HD 85567 is consistent with the hypothesis that it is a YSO.

We then investigate why HD 85567 appears undersized according to the size luminosity relationship of YSOs. The size of the $K$-band emitting region is congruous with the predicted location of the dust sublimation assuming an accretion disc that is optically thick in the inner regions. Furthermore, the differential phase observations over the $\mathrm{CO}$ bandhead are also consistent with a compact ( $r \sim 1 \mathrm{AU}$ ) gaseous disc interior to the dust disc. More extended discs do not reproduce the data as well.

To conclude, the data support the hypothesis that HD 85567 appears undersized according to the YSO size luminosity relationship due to the presence of an optically thick gaseous disc interior to the dust sublimation radius. This indicates that HD 85567 is indeed a YSO. If this is the case, the gaseous inner disc may be identified as an accretion disc. The presence of an optically think inner disc and the absence of a far infrared excess suggest that HD 85567 is photo-evaporating its disc from the outer edge.

Acknowledgements. HEW acknowledges the financial support of the Max Planck Society. This research has made use of the AMBER data reduction package of the Jean-Marie Mariotti Center. This publication also makes use of VOSA, developed under the Spanish Virtual Observatory project supported from the Spanish MICINN through grant AyA2008-02156.

\section{References}

Acke, B., van den Ancker, M. E., \& Dullemond, C. P. 2005, A\&A, 436, 209 Alonso-Albi, T., Fuente, A., Bachiller, R., et al. 2009, A\&A, 497, 117

Baines, D., Oudmaijer, R. D., Porter, J. M., \& Pozzo, M. 2006, MNRAS, 367, 737

Bayo, A., Rodrigo, C., Barrado, Y., et al. 2008, A\&A, 492, 277

Beichman, C. A., Neugebauer, G., Habing, H. J., Clegg, P. E., \& Chester, T. J. 1988, Infrared astronomical satellite (IRAS) catalogs and atlases (Washington, DC: NASA RP-1190), 1

Benisty, M., Tatulli, E., Ménard, F., \& Swain, M. R. 2010, A\&A, 511, A75

Bonneau, D., Clausse, J.-M., Delfosse, X., et al. 2006, A\&A, 456, 789

Bonneau, D., Delfosse, X., Mourard, D., et al. 2011, A\&A, 535, A53

Cardelli, J. A., Clayton, G. C., \& Mathis, J. S. 1989, ApJ, 345, 245

Chelli, A., Utrera, O. H., \& Duvert, G. 2009, A\&A, 502, 705

Cutri, R. M., \& et al. 2012, VizieR Online Data Catalog, II/311

de Winter, D., van den Ancker, M. E., Maira, A., et al. 2001, A\&A, 380, 609

DENIS Consortium 2005, VizieR Online Data Catalog, II/263

Dullemond, C. P., \& Monnier, J. D. 2010, ARA\&A, 48, 205

Eisner, J. A., Lane, B. F., Akeson, R. L., Hillenbrand, L. A., \& Sargent, A. I. 2003, ApJ, 588, 360 
Eisner, J. A., Lane, B. F., Hillenbrand, L. A., Akeson, R. L., \& Sargent, A. I. 2004, ApJ, 613, 1049

Eisner, J. A., Chiang, E. I., Lane, B. F., \& Akeson, R. L. 2007, ApJ, 657, 347

Eisner, J. A., Graham, J. R., Akeson, R. L., \& Najita, J. 2009, ApJ, 692, 309

Eisner, J. A., Monnier, J. D., Woillez, J., et al. 2010, ApJ, 718, 774

Gai, M., Menardi, S., Cesare, S., et al. 2004, in SPIE Conf. Ser. 5491, ed. W. A. Traub, 528

Helou, G., \& Walker, D. W. 1988, Infrared astronomical satellite (IRAS) catalogs and atlases, The small scale structure catalog, 7

Herbig, G. H. 1960, ApJS, 4, 337

Høg, E., Fabricius, C., Makarov, V. V., et al. 2000, A\&A, 357, 367

Ilee, J. D. 2013, Ph.D. Thesis, University of Leeds UK

Ishihara, D., Onaka, T., Kataza, H., et al. 2010, A\&A, 514, A1

Klare, G., \& Neckel, T. 1977, A\&AS, 27, 215

Kraus, S., Hofmann, K.-H., Benisty, M., et al. 2008a, A\&A, 489, 1157

Kraus, S., Preibisch, T., \& Ohnaka, K. 2008b, ApJ, 676, 490

Kraus, S., Hofmann, K., Malbet, F., et al. 2009, A\&A, 508, 787

Kreplin, A., Kraus, S., Hofmann, K.-H., et al. 2012, A\&A, 537, A103

Lamers, H. J. G. L. M., Zickgraf, F.-J., de Winter, D., Houziaux, L., \& Zorec, J. 1998, A\&A, 340, 117

Malbet, F., Benisty, M., de Wit, W., et al. 2007, A\&A, 464, 43

Malfait, K., Bogaert, E., \& Waelkens, C. 1998, A\&A, 331, 211

Meeus, G., Waters, L. B. F. M., Bouwman, J., et al. 2001, A\&A, 365, 476

Mermilliod, J.-C., \& Mermilliod, M. 1994, Catalogue of Mean UBV Data on

Stars, VI (Berlin Heidelberg New York: Springer-Verlag), 1387

Millan-Gabet, R., Schloerb, F. P., Traub, W. A., et al. 1999, ApJ, 513, L131

Millan-Gabet, R., Schloerb, F. P., \& Traub, W. A. 2001, ApJ, 546, 358
Miroshnichenko, A. S., Levato, H., Bjorkman, K. S., \& Grosso, M. 2001, A\&A, 371,600

Monnier, J. D., \& Millan-Gabet, R. 2002, ApJ, 579, 694

Monnier, J. D., Millan-Gabet, R., Billmeigrf, E. A., et al. 2005, ApJ, 624, 832

Monnier, J. D., Berger, J.-P., Millan-Gabet, R., et al. 2006, ApJ, 647, 444

Murakami, H., Baba, H., Barthel, P., et al. 2007, PASJ, 59, 369

Natta, A., Prusti, T., Neri, R., et al. 2001, A\&A, 371, 186

Onaka, T., Matsuhara, H., Wada, T., et al. 2007, PASJ, 59, 401

Renard, S., Malbet, F., Benisty, M., Thiébaut, E., \& Berger, J. 2010 A\&A, 519, A26

Schild, R. E., Garrison, R. F., \& Hiltner, W. A. 1983, ApJS, 51, 321

Skrutskie, M. F., Cutri, R. M., Stiening, R., et al. 2006, AJ, 131, 1163

Tannirkulam, A., Monnier, J. D., Millan-Gabet, R., et al. 2008, ApJ, 677, L51

Tatulli, E., Isella, A., Natta, A., et al. 2007a, A\&A, 464, 55

Tatulli, E., Millour, F., Chelli, A., et al. 2007b, A\&A, 464, 29

Thé, P. S., de Winter, D., \& Perez, M. R. 1994, A\&AS, 104, 315

Verhoeff, A. P., Waters, L. B. F. M., van den Ancker, M. E., et al. 2012, A\&A, 538, A101

Vink, J. S., Drew, J. E., Harries, T. J., \& Oudmaijer, R. D. 2002, MNRAS, 337, 356

Vinković, D., \& Jurkić, T. 2007, ApJ, 658, 462

Weigelt, G., Grinin, V. P., Groh, J. H., et al. 2011, A\&A, 527, A103

Wheelwright, H. E., Oudmaijer, R. D., de Wit, W. J., et al. 2010, MNRAS, 408, 1840

Wheelwright, H. E., de Wit, W. J., Weigelt, G., Oudmaijer, R. D., \& Ilee, J. D. 2012, A\&A, 543, A77

Wright, E. L., Eisenhardt, P. R. M., Mainzer, A. K., et al. 2010, AJ, 140, 1868

Pages 9 to 10 are available in the electronic edition of the journal at http://www . aanda. org 


\section{Appendix A: Transfer functions}

An example of the transfer functions at a particular wavelength is presented in Fig. A.1. It can be clearly seen that in two cases (2012/04/06 and 2013/03/04), the visibilities of HD 85567 are the same or similar to the calibrators, indicating a compact source. In the other two cases (2012/05/06 and 2012/12/29), the visibilities of HD 85567 are noticeably lower than the calibrators, indicating a resolved source.
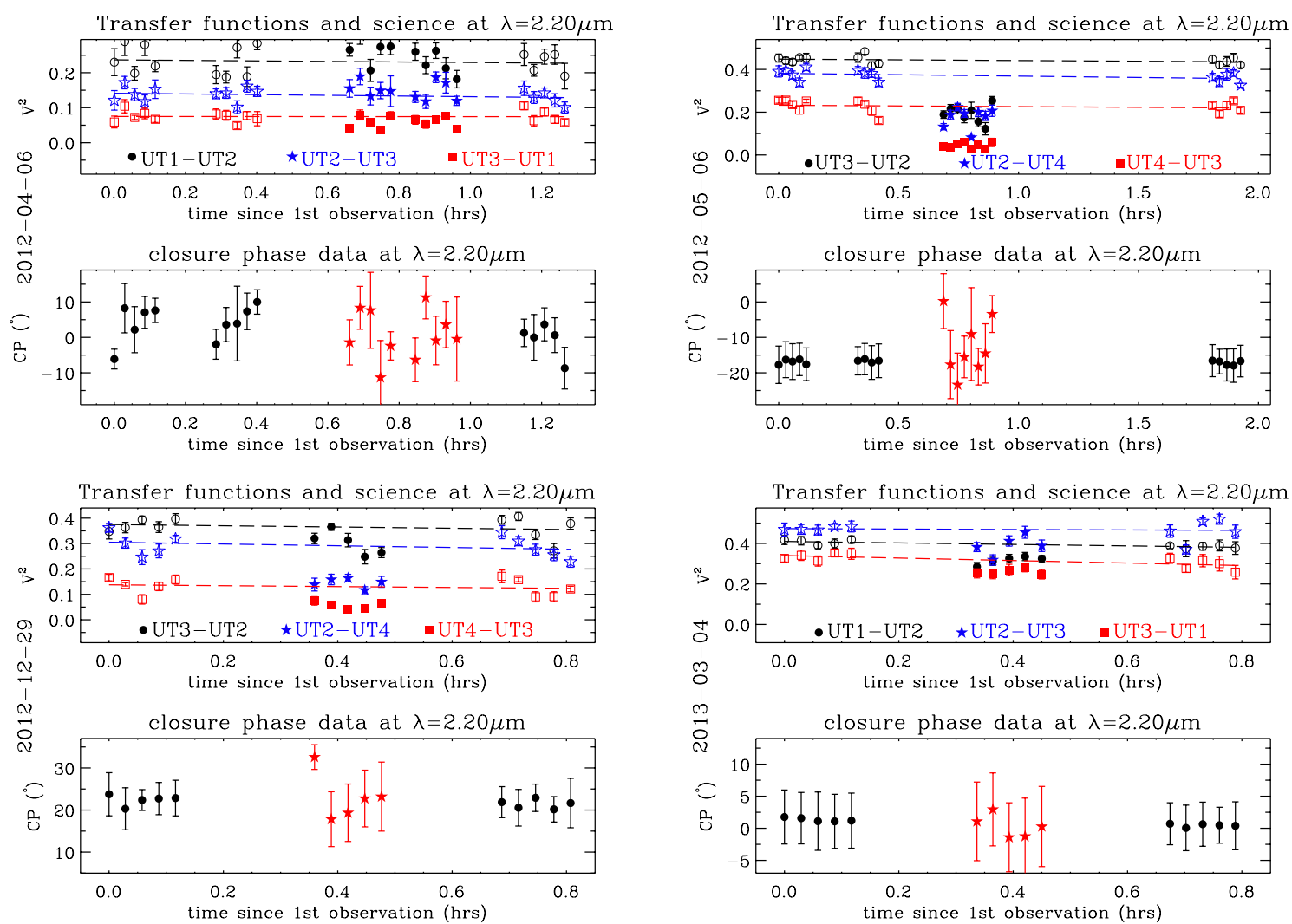

Fig. A.1. Examples of the transfer functions and raw science data at a wavelength of $\lambda=2.2 \mu \mathrm{m}$. In the visibility plots, open symbols mark calibrator observations and filled symbols mark the observations of HD 85567. In the closure phase plots, the observations of HD 85567 are marked by star symbols and calibrator data by filled circles. A frame selection of 10 percent was used, but the behaviour is independent of the frame selection used. 

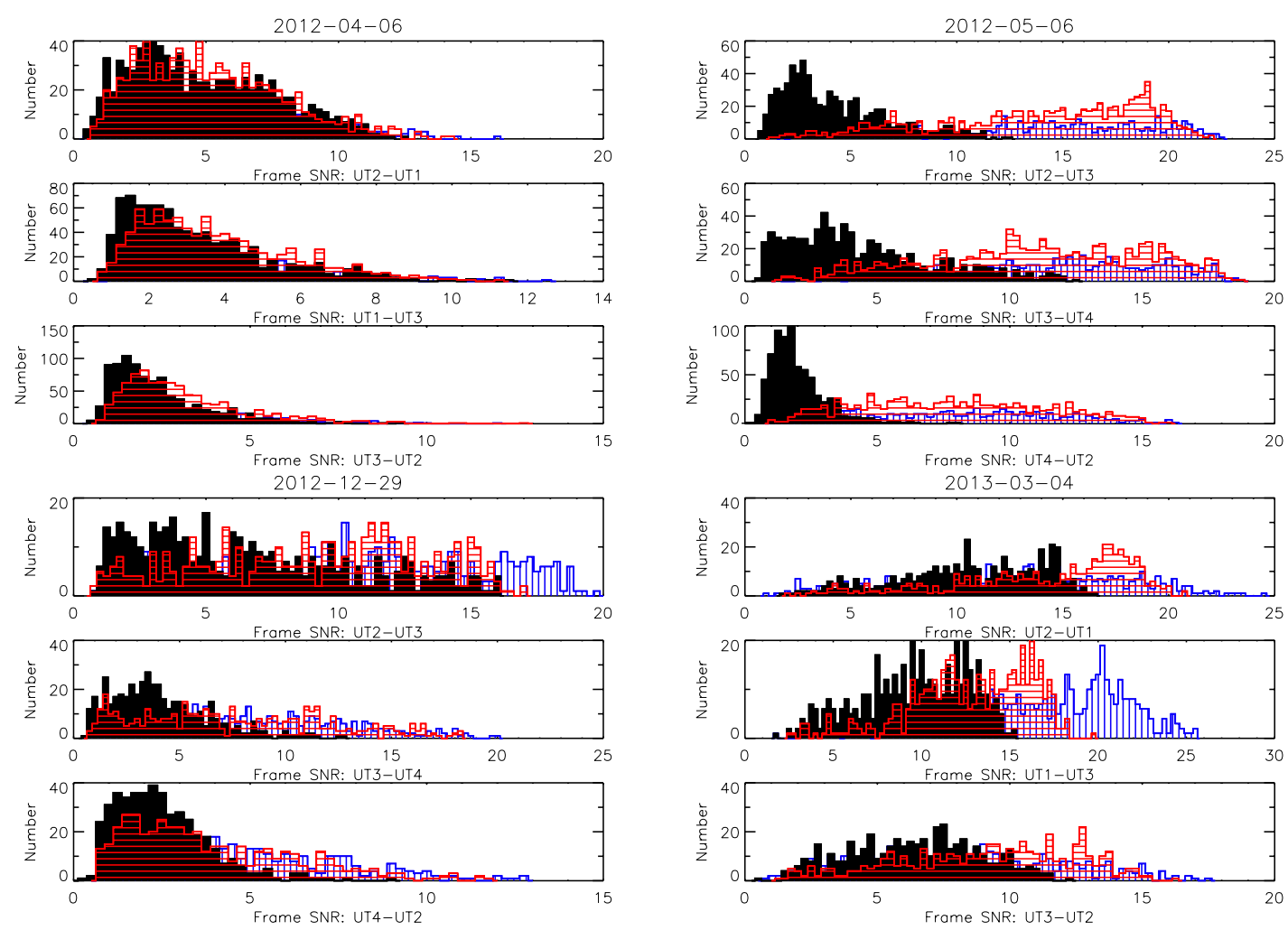

Fig. B.1. Fringe $\mathrm{S} / \mathrm{N}$ distributions for each observing date and each baseline. The filled black histogram is the fringe $\mathrm{S} / \mathrm{N}$ distribution of the observations of HD 85567. The distributions filled with vertical blue and horizontal red lines mark the fringe S/Ns associated with the calibrators HD 85313 and HD 84177 respectively.

\section{Appendix B: Fringe S/N distributions}

The distribution of the fringe $\mathrm{S} / \mathrm{Ns}$ of the science and calibrator observations are presented in Fig. B.1. The two cases where HD 85567 appears resolved (2012-05-06 and 2012-12-29) are considered first.

It can be seen that in these cases, the $\mathrm{S} / \mathrm{N}$ distributions of the calibrators extend to significantly higher values than the distribution of the HD 85567 observations. This is particularly apparent in the case of the observations conducted on 2012-05-06. On this occasion, the fringe S/N distribution of HD 85567 on the UT2-UT3 baseline peaks at $\sim 2$ while the HD 84177 distribution has a skewed distribution peaking at $\sim 18$. This disparity could be due to the fact that although the $K$-band magnitudes of the target and calibrators are similar, HD 85567 is 1 magnitude dimmer than the calibrators in the $H$-band. The fringe tracking is performed in the $H$-band. Therefore, the performance of the fringe tracking was likely higher when observing the calibrators than when observing the target. Furthermore, the observations of the target were associated with worse seeing than the calibrator observations. This could also reduce the fringe tracking performance. Consequently, the high $\mathrm{S} / \mathrm{N}$ of the calibrator observations is likely due the fringe tracking performing best when observing the calibrators. This hypothesis is substantiated by the FINITO data recorded by the RMNREC software. In the two cases where the target visibilities appear significantly lower than those of the calibrators, the rms of the FINITO phases associated with the target is up to 40 percent larger than that associated with calibrator observations.

The case of the two dates when the target appears close to unresolved (2012-04-06 and 2013-03-04) are now discussed.

In the case of the observations conducted on 2012-04-06, it can be seen that the target and calibrator fringe $\mathrm{S} / \mathrm{N}$ distributions are almost identical. This suggests that the calibration is accurate. However, as can be seen, the typical S/Ns are relatively low; all the distributions peak below 5 . Therefore, these observations are relatively noisy. It can be seen that the observations on 201303-04 are of higher quality with typical S/Ns of approximately 10. In this case, the target and calibrator distributions are not identical but there is considerable overlap, which, in conjunction with the high $\mathrm{S} / \mathrm{N}$, suggests that calibration in this case should be reliable. The superior S/N of the HD 85567 observations conducted on 2013-03-04 is attributed to the AMBER intervention of January 2013 which improved the sensitivity of AMBER, particularly in the $H$-band where fringe tracking is conducted.

The disparity between the fringe $\mathrm{S} / \mathrm{N}$ distributions of the calibrator and science observations of 2012-05-06 and 2012-12-29 could result in an inaccurate calibration. This could occur in the following manner. The lower fringe $\mathrm{S} / \mathrm{N}$ of the target observations could result in the target fringes exhibiting a lower fringe contrast than the calibrators, thus making the target appearing more resolved than the calibrators, even if this is not the case. Indeed, as noted above, the raw visibilities of HD $85567 \mathrm{ob}-$ tained on these two dates are lower than those of the calibrators. We surmise that the difference between the target and calibrator observations on these dates is a bias caused by the fringe tracking performance degrading when observing the target.

We conclude that only the observations when the fringe $\mathrm{S} / \mathrm{N}$ distributions of the target and calibrator observations are similar can be calibrated. In principle, the observations of 2012-0406 and 2013-03-04 offer reliable calibration. However, since the fringe S/Ns of the observations conducted 2012-04-06 are relatively low, this paper focuses exclusively on the observations obtained on the date 2013-03-04. 